\title{
The cooperative human
}

\author{
Human beings are a social species that relies on cooperation to survive and thrive. Understanding how and \\ why cooperation succeeds or fails is integral to solving the many global challenges we face.
}

C ooperation lies at the heart of human lives and society - from day-to-day interactions to some of our greatest endeavours. Understanding cooperation - what motivates it, how it develops, how it happens and when it fails to happen - is therefore an important part of understanding all kinds of human behaviour. In this focus issue of Nature Human Behaviour, we bring together review, opinion and research content on human cooperation from across the journal's scope - including evolution, anthropology, ecology, economics, neuroscience and environmental science to spark interdisciplinary conversation and perhaps even inspire some scientific cooperation. In our dedicated collection on cooperation (https://www.nature.com/ collections/gvmywthghh), we combine new commissioned content with work from our archive that exemplifies the breadth of enquiry in this important interdisciplinary field.

Why do we cooperate at all, when choosing the selfish option may seem like the most logical and rewarding in a competitive world? A Review by Hilbe et al. discusses the recent formal theoretical work on partnership versus rivalry in social dilemmas and argues that rivalry tends to develop in smaller populations with limited numbers of interactions, while more frequent interactions encourage cooperation to emerge as an evolutionarily stable strategy. Modelling work by Jagau and van Veelen ${ }^{1}$ we published last year also found evidence for multiple stable states of cooperation, showing, in contrast to previous work, how flexible, deliberative strategies can evolve. Of course, many other species have made a success out of cooperation, and a Comment by Brosnan makes the case for a comparative economics approach to uncover cooperation's evolutionary path.

We can also interrogate the mechanisms and motives behind cooperation by observing how it happens in practice. In a Review, Fehr and Schurtenberger evaluate the experimental literature for evidence of a fixed social norm of conditional cooperation supported by peer punishment, which, they argue, can account for multiple recurring patterns of human behaviour seen in cooperative contexts. In future, we may gain an even more precise understanding of individual motives in these types of cooperative experiments, by directly observing neural responses during game play, as argued in a Comment by Declerck and colleagues.

Successful cooperation requires not only cooperative choices, but also a way to signal your intent and good qualities to potential partners. A Perspective by Bliege-Bird and colleagues examines the subtle signalling that people deploy to solidify their long-term cooperative relationships, using the practice of sharing catches after lizard hunts among Martu hunter-gatherer women as a case study. The most successful hunters share out, subtly, the meat with all, strengthening their reciprocal bonds and distributing the burden of resource scarcity risks. This chimes with a Comment by Aktipis et al., who suggest that the evolutionary concept of 'fitness interdependence' between individuals for survival and reproduction could be adopted as a framework across disciplines to understand why cooperation is so integral to our lifeways.

\section{Even in difficult situations, the desire for cooperation would appear to often be nascent and the evidence suggests that we are naturals at it, given the opportunity.}

The Martu example is a system of trust and shared risk that speaks to some of the most pressing issues that humans face today globally, that is, the sharing of risk and the 'trust' needed to take collective action. In a previous issue, Koomen and Herrmann $^{2}$ showed that children as young as six years old can spontaneously find ways to collaborate to maintain a shared, limited resource. And indeed, a 2017 review of the literature by McAuliffe et al. ${ }^{3}$ provided ample evidence that children acquire notions of fairness from a surprisingly early age. However, we know all too well from observing the real world that coordination among adults often fails. Gächter et al. ${ }^{4}$ provided partial insight into why this might be, showing that adult participants contribute more when establishing a new

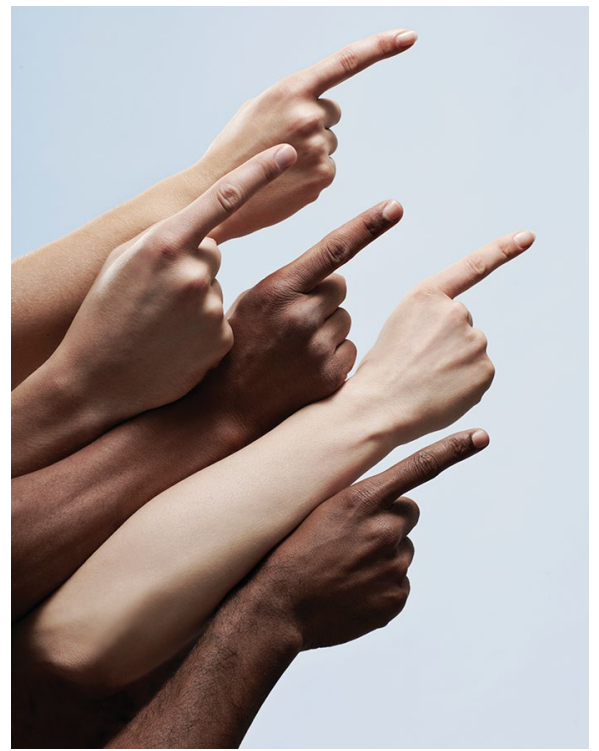

Credit: Klaus Vedfelt/DigitalVision/Getty

collective good, but contribute much less to maintain an existing resource. Muthukrishna et al..$^{5}$ showed that typical anti-corruption strategies may have negative impacts on cooperation, depending on the cultural context. These findings are a caution that the levers we apply to encourage cooperation should be tailored to context.

How can we nurture cooperation for the common good? In experiments run by Grossmann et al. ${ }^{6}$, the authors found that they could induce participants to engage in 'wise reasoning' to avert making automatic, selfish decisions. In a Comment, Dannenberg and Barrett note that cooperation often fails when individuals are uncertain about the relative importance of their own effect on a critical, environmental threshold, and argue, through successful examples, such as the Montreal Protocol, that institutions must make cooperation the more attractive option. In a similar vein, Castilla-Rho et al. presented a model that identifies the 'tipping points' at which groundwater conservation becomes a widely accepted social norm across diverse cultural settings, which can be used by conservation managers to predict the most effective interventions.

Finally, some of the seemingly most intractable cooperation problems in the world today are the conflicts between rival nations and different political, religious 
or ethnic groups. However, Fotouhi et al. show that a strong barrier to cooperation is simple lack of communication and suggest that promoting even sparse interconnections between previously segregated societies can support the evolution of cooperation globally. Even in difficult situations, the desire for cooperation would appear to often be nascent and the evidence suggests that we are naturals at it, given the opportunity.

Some powerful theories and empirical insights have expanded our knowledge of cooperation over the past few decades but much remains to be understood. Integrating questions and approaches from different fields may provide fertile ground to achieve this. We look forward to publishing theoretical and empirical research on cooperation in the future that will push the boundaries of the field even further.

Published online: 9 July 2018

https://doi.org/10.1038/s41562-018-0389-1
References

1. Jagau, S. \& van Veelen, M. Nat. Hum. Behav. 1, 0152 (2017).

2. Koomen, R. \& Herrmann, E. Nat. Hum. Behav. 2, 348-355 (2018).

3. McAuliffe, K., Blake, P. R., Steinbeis, N. \& Warneken, F. Nat. Hum. Behav. 1, 0042 (2017).

4. Gächter, S., Kölle, F. \& Quercia, S. Nat. Hum. Behav. 1, 650-656 (2017).

5. Muthukrishna, M., Francois, P., Pourahmadi, S. \& Henrich, J. Nat. Hum. Behav. 1, 0138 (2017).

6. Grossmann, I., Brienza, J. P. \& Bobocel, D. R. Nat. Hum. Behav. 1 0061 (2017).

7. Castilla-Rho, J. C., Rojas, R., Andersen, M. S., Holley, C. \& Mariethoz, G. Nat. Hum. Behav. 1, 640-649 (2017). 